International Journal of Industrial Engineering
and Operations Management (IJIEOM)
Volume 3, No. 2, December 2021
pp. $58-74$
$\begin{gathered}\text { IEOM Society } \\ \text { International }\end{gathered}$

\title{
Maximum Coverage Location Model for Fire Stations with Top Corporate Risk Locations
}

Running Title: MCLP for Fire Stations with Top Corporate Risk Locations

\author{
${ }^{1}$ Abdulaziz Saleh Alzahrani, ${ }^{2}$ Ahmad Al Hanbali \\ King Fahd University of Petroleum \& Minerals Ringgold standard institution - System Engineering, \\ Dhahran, Saudi Arabia \\ abdulaziz.saleh0@gmail.com, ahmad.alhanbali@kfupm.edu.sa \\ https://doi.org/10.46254/j.ieom.20210201
}

\begin{abstract}
The fire station location is a critical decision to optimize the coverage level as measured in terms of the response time. This paper focuses on optimizing the coverage problem, especially in the fire protection field, with new model features to incorporate realistic business challenges such as location criticality and secondary coverage. We extend the deterministic Maximum Coverage Location Problem to account for Top Corporate Risk locations being covered by different fire stations as primary and secondary coverage. To deal with the response time uncertainty arising in practice, we propose a new binary linear problem based on the Maximum Expected Covering Location Problem. By exploiting the model structural characteristics, we prove that the model complexity can be substantially reduced to yield an efficient solution. In the numerical experiments, we use a real case study with five years of historical data. The optimization results of the models yield a priority ranking of the fire stations to open and show the value of incorporating the coverage uncertainty. Finally, we also compare our model with uncertainty with the standard scenario-based optimization to extend the numerical results.
\end{abstract}

ARTICLE INFO

Submitted

Feb 28, 2021

Revised

June 20, 2021

Accepted

July 29,2021

\section{KEYWORDS}

Fire Station Location; Primary Coverage;

Secondary Coverage; Maximum Covering Location Problem; Uncertainty modelling

\section{Introduction}

Fire departments are being challenged to optimize operating costs while maintaining an adequate level of service. Optimizing the operating cost of an existing network of fire stations is limited to improvement to either the firefighters (crew size) or fire apparatus (trucks/engines). Effectively, the optimization is constrained by the existing locations since the time to respond to an incident depends mainly on them and on the incident sites. Therefore, locating a fire station is critical to optimize the service level and ultimately ensure optimized operating costs. In this paper, we shall be limited to the location decision. The decision on the fire station location is made based on input parameters such as the coverage area, nearby fire stations, and the required response time. Before locating fire stations, all these inputs are considered to determine the standard of response coverage. That is, the policy to establish effective and timely coverage within the area of concern. The standard of response coverage is encapsulated into one measure, namely the total response time. In the US, the NFPA (National Fire Protection Association) issues general recommendations for total response time, and this standard is known as (NFPA 1710, 2020). The overall response time in NFPA 1710 is measured as the time elapsed from answering an alarm to the arrival of full response force (personnel and equipment) to the incident scene. The total response time varies depending on the type of incident location, but the general guideline is between 8 to 10 minutes. 
Based on the risk assessment conducted at the company of our case study, a 5-minutes response time target is set for a community area and a 10-minutes for the industrial area. Such response times are referred to as primary response times, and in practice, they are treated as KPIs to be met by each fire station. Generally, the primary response time can be defined as the time needed for the arrival of a full response force; however, an equally important measure is the time for the first response force arrival. The difference between community and industrial zone response time stems from the strategy to approach each type of fire incident. For example, the strategy for community fires is to attack and be aggressive to avoid the possibility of a loss of life. In contrast, the strategy for industrial fires is to contain the fire and limit its exposure. In addition to the primary response time, the secondary response time is equally important, which is defined as the time elapsed from alarm answering to the arrival of supporting fire personnel. Generally, the secondary response time is defined as a form of KPI by the supporting fire station.

In this paper, we address the fire stations location within a large company complex in Saudi Arabia. The complex covers a total area of 80 square $\mathrm{km}$ and has a population of around 37,000 people. The complex comprises of industrial facilities, office buildings, housing areas, a medical facility, and schools, among other amenities. Currently, the company has two fire stations with different response capabilities. The company considers adding more stations or replacing the existing stations to provide an adequate response to all areas within the complex. Therefore, the company objective is to determine the required number and locations of the fire stations to meet the response time targets for each incident type and consider location criticality. The company's complex has 134 locations where a subset of those locations is identified as Top Corporate Risk (TCR) locations. As an example, the health and R\&D centers are classified as TCR locations. The TCR locations must be covered by more than one station with a predefined secondary response time of no more than 15 minutes. The rationale behind having 15 minutes as secondary response time is that when responding to incidents, continuous deployment of attack lines is required, and each attack line requires breathing apparatus that lasts about 1520 minutes. At the company, the adherence to the primary and secondary response are well-defined performance measures and are closely monitored by the management. From a corporate perspective, location criticality is of paramount importance when locating fire stations. Ten locations are identified as critical and described as the top ten corporate risk locations for the company. To determine the TCR location criticality weight, four experienced fire professionals at the company were interviewed to perform a pairwise comparison among the different TCR locations.

The objective is to determine how many fire stations are needed and where to locate them to ensure a specified coverage time target for a set of incident locations. This paper provides several realistic extensions to the seminal model in this research area, well-known as the Maximum Covering Location Problem (MCLP) (Church and ReVelle 1974). The model aims to maximize the covered locations within a specified target (time or distance) subject to a fixed number of facilities (fire stations). The problem has been proven to be NP-hard by Megiddo et al. (1983), and consequently, the extensions considered here are also NP-hard. Daskin (1983) was among the first to include randomness in the coverage problem in the Maximum Expected Covering Location Problem (MEXCLP). To the best of our knowledge, our case study specific features were not considered in the literature. Therefore, in this paper, we extend several existing models to meet the company requirements and objectives. Namely, we extend the MCLP model to ensure the coverage of TCR locations while considering varying the required number of fire stations and allowing the secondary coverage for those critical locations. Moreover, two versions of the model are proposed to account for uncertainty. The uncertainty type of interest here is the response time uncertainty. The first version deals with uncertainty through scenario-based optimization, and the second version is an extension (MEXCLP) presented by Daskin (1983). The contributions in this paper can be summarized as follows:

We extend the maximum coverage location problem (MCLP) to account for TCR locations covered by different fire stations as primary coverage and secondary coverage.

We broaden the relevancy of the Maximum Expected Covering Location Problem (MEXCLP) by relaxing its strict assumption on the coverage probability. This is done by considering the coverage probability as a function of the fire station and the incident location. We also provide an efficient formulation with a substantial complexity reduction for this extension.

We apply the model to a real case study consisting of five potential fire stations, 134 incident locations, ten TCR locations, and using five years of fire incidents data to estimate the uncertainty parameters.

This paper is organized as follow. In Section 2, we survey the literature and showcase the development of several coverage models and their applications. Section 3 provides the research methodology used and the extended MCLP, including the model formulation of the deterministic case and the stochastic case. In Section 4, we solve a real case 
study for a large company complex in Saudi Arabia. In Section 5, we discuss and compare the results of the different models. The last section includes some final remarks and directions for future research.

\section{Literature Review}

This paper provides several realistic extensions to the Maximum Covering Location Problem (MCLP) initially introduced by Church and ReVelle (1974). The original model aims to maximize the covered demand within the desired service target (time or distance) by deciding on the facilities' location to be opened from a given subset of potential locations. This work extends the original problem by considering two main extensions: the first extension is having a subset of locations that must be covered regardless of the number of opened fire stations, and the second extension is considering secondary coverage. It is worth noting that the definition of secondary coverage, sometimes referred to as backup or double coverage, is slightly different from the definition found in the published literature, where it refers to covering a location twice within the same response time. The secondary coverage is defined here as at least two fire stations, including the primary coverage, must cover a location within a secondary response time of 15 minutes. A new stochastic version for the extended deterministic model is considered based on the Maximum Expected Covering Location Model (MEXCLP). Therefore, this section covers recent publications in: (1) Maximum Coverage Location problems, and (2) Coverage problems that account for uncertainty.

\subsection{Maximum Coverage Location Problems}

Coverage location models for emergency response and fire stations have been widely studied in the literature. The most recent comprehensive literature review for the coverage problems was presented by Farahani et al. (2012). The authors provided classifications, used solutions, and application areas of coverage models. To the best of our knowledge, the first model relating to locating the fire stations was introduced by Toregas et al. (1971). The model's objective is to cover all demand nodes, known as Location Set Covering Problem (LSCP). The model was a basic set covering, resulting in more fire stations (facilities) than needed since all demand nodes must be covered regardless of their size and importance. From a practical perspective, the decision-maker may prefer to see what happens if the coverage is below $100 \%$. Such need has led to the Maximum Covering Location Problem (MCLP) development, which was introduced by Church and ReVelle (1974). The model aims to maximize the covered demand within the desired service target (time or distance) by deciding on the facilities location to open from a given subset of potential locations. The MCLP model has been further extended to allow for multiple levels of backup, such as the work of Hogan and ReVelle (1986), Pirkul and Schilling (1989), and Narasimhan et al. (1992). Some authors relaxed the assumption of a full coverage to allow for partial coverage where the distance is assumed to be a range rather than a single value (Karasakal \& Karasakal, 2004). Çatay (2011) developed a model to locate fire stations in the city of Istanbul and considered multiple levels of coverage, i.e., covered by more than two stations within the target response time. The model accounts for the risk category of each demand region while incorporating different response times, depending on each region's risk category. Aktaş et al. (2013) determined the locations for additional fire stations in Istanbul by considering a 5-minute response time to incidents. The authors used the Geographic Information System (GIS) to model the Istanbul road network and solved two models (set covering and maximal covering) to optimality. Ulander (2015) presented an optimization model that locates fire and rescue resources to meet the expected demand to minimize the response time for different incident types. Ozbaygin et al. (2016) presented an extension to MCLP to allow for partially covering a demand point if it is close enough to a covered demand by a given tour. The authors integrated the MCLP with the routing problem and proposed an algorithm to solve the model. Based on Murray (2016), "With more than 1,500 citations in the academic literature, MCLP has truly stood the test of time and may actually be as relevant or more so today". MCLP is a binary linear problem and was proven to be NP-hard by Megiddo et al. (1983) and hence the extensions considered here are also NP-hard. Therefore, several heuristics and algorithms have been proposed to solve the MCLP Church and ReVelle (1974) and Cordeau et al. (2019).

\subsection{Coverage Problems with Uncertainty}

The models covered in the above review deals with deterministic cases where the optimization assumes all parameters are known and deterministic. However, many practical applications include unknown and uncertain parameters. In such cases, stochastic programming can be applied to model the uncertainty. The reader is referred to the tutorial made by Shapiro and Philpott (2007) for an overview of the different methods through which uncertainty can be modelled. For the coverage problem, the probability of a facility to respond to an incident might be uncertain, and possibly the response time could be uncertain. Therefore, the uncertainty of such parameters needs to be included in the model. 
To the best of our knowledge, the first coverage model considering uncertainty was presented by Daskin (1983), coined as Maximum Expected Covering Location Problem (MEXCLP). The MEXCLP model extended the original MCLP model to account for the probability that facilities may not be able to cover a demand when it receives a fire alarm. The model considers the same probability of being busy, not be able to respond, for all sites; therefore, it is independent of the facility site. Moreover, the probability of one facility being busy is independent of other facilities. Aytug and Saydam (2002) reformulated the MEXCLP as a non-linear model and proposed multiple genetic algorithms to solve the MEXCLP. The algorithms performed better in terms of solution time; however, the quality of the solutions was not substantially better than the algorithm of Daskin (1983).

In other papers modelling uncertainty such as the work of Daskin et al. (1988), the authors integrated MEXCLP with multiple coverage levels and analysed their linkage. Davari et al. (2011) extended the MCLP model to account for uncertainty using fuzzy travel times and proposed a simulated annealing approach to solving the extended model with high accuracy. Ven den Berg et al. (2016) presented a linearized version of MEXCLP, which allows partial coverage in contrast to Daskin (1983), where full coverage is assumed. Such assumptions were explored earlier by Ingolfsson et al. (2008) and Erkut et al. (2008) but with the non-linear version of the problem. Abdul Ghani and Ahmad (2017) used a Monte Carlo Simulation to estimate the uncertainty in travel times for several coverage models. Dreyfuss and Giat (2017) considered a less-restrictive version of the coverage problem with the application to maintenance and repair system where the waiting time for failed items is relaxed to have a time window rather than an expectation. Due to the problem's nature in this paper, such relaxation is not considered as fires typically intensify with time passage, and the objective is to respond and contain the fire.

Based on the above discussion, the models presented in this paper have new and unique features. The new models consider a weighted maximum coverage location problem while ensuring that a set of locations (identified as TCR locations) must be covered. Besides, the company would like to ensure secondary coverage with secondary response time for the TCR locations. Also, the assumption of independent coverage probability is relaxed to have the probability as a function of the facility site and the incident location. As far as we know, these have not been addressed before.

\section{Research Methodology}

In this paper, we use the mathematical programming modelling approach to solve the coverage problem of the case study to optimality while satisfying all the company requirements on primary and secondary coverage of the TCR locations. In Figure 3.1, we give a flowchart of the process of incident response, the model formulation and results. In section 3.1, we present a deterministic version of the problem and refer to it as the weighted maximum coverage location problem with secondary coverage for top corporate risk. Section 3.2 presents a stochastic version of the problem that takes the coverage uncertainty into account and refers to it as the maximum expected covering location model with secondary coverage for top corporate risk. Different solution sets are generated for both models based on the selected number of fire stations to be opened, and the associated coverage gained is reported. These results help the decision-maker in determining the solution set to be selected. We shall follow the standard approach for the models explanation, starting with the definitions of indices, input variables, and decision variables, and then giving the model objective function and constraints. The input variables used are collected from the case study considered in this paper; for more details, see Section 4.1.

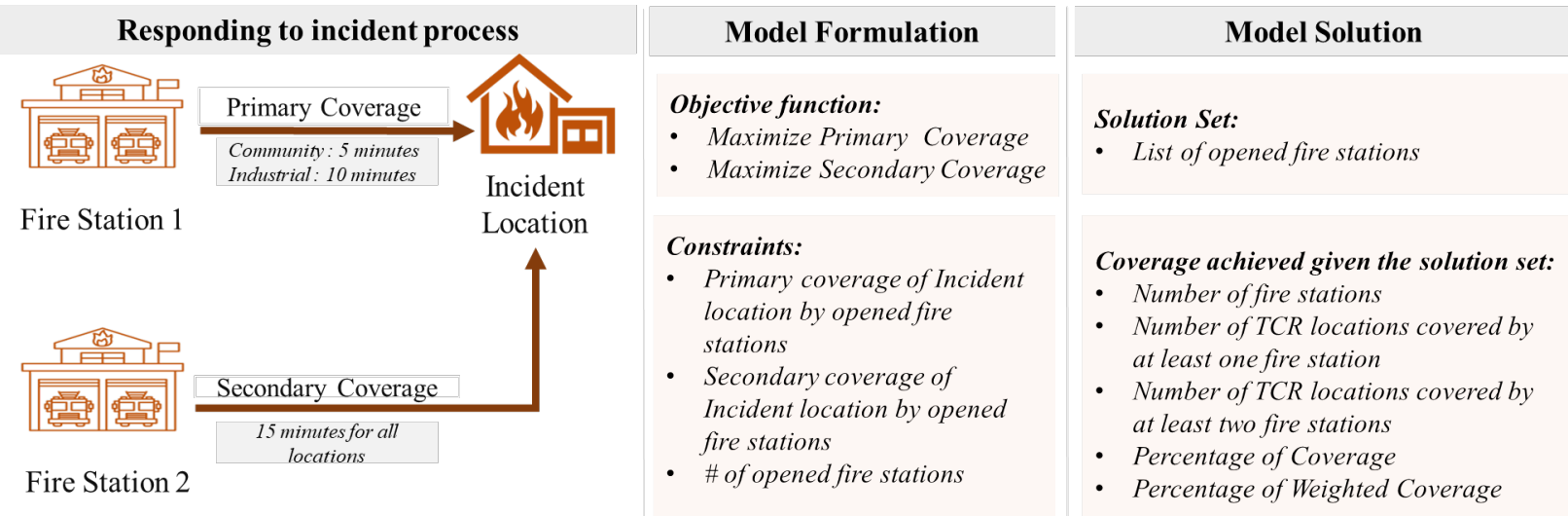

Figure 3.1: Flowchart for responding to fire incident process, model formulation and solution 


\subsection{Weighted Maximum Coverage Location Problem with Secondary Coverage for Top Corporate Risk (WMCLP-STCR)}

Different coverage models are required depending on each decision maker's objectives from the type and level of coverage to the risk attitude. In the current problem, the TCR locations must be covered by more than one station with no more than 15 minutes as response time. When responding to incidents, continuous deployment of attack lines is required, and each attack line requires a breathing apparatus that lasts about 15 to 20 minutes. Also, if the fire station responsible for the primary coverage was unsuccessful in controlling the fire, then the other station helps supply additional attack lines as a form of secondary coverage. As a result, the fire department has set 15 minutes as a secondary response time target, and it wants to explore possible coverage based on the number of fire stations opened. The MCLP model would need to be extended to account for the company's requirements. The first requirement is to ensure at TCR locations are covered twice, which means the minimum number of fire stations to be opened must be two or more stations, and to ensure primary coverage and secondary coverage for all TCR locations. It is worth noting that secondary coverage usage in this work is different from the typical definition found in the literature, see Section 2.1. We define it as a separate time target (higher than the primary coverage time target) that must be met by at least two fire stations. The model objective would need to be modified to maximize the coverage of all locations along with ensuring TCR locations are primarily and secondarily covered. TCR locations differ in their criticality as voiced by the subject matter expert. To that end, we have interviewed four experienced fire professionals at the company to determine the weights (refer to Section 4.1 for the details). Therefore, the model of interest to the company complex under study is the Weighted Maximum Coverage Location Problem with Secondary Coverage for Top Corporate Risk (WMCLP-STCR).

\section{WMCLP-STCR Problem formulation}

To formally state the problem, we consider the following notations:

Indices:

$i=$ index of location sites (where $i=1,2 \ldots N)$

$j=$ index of fire station sites (where $j=1,2 \ldots M$ )

$k=$ index of location sites that are considered as Top Corporate Risk (where $k=1,2 \ldots R$ and $R \leq N$ ).

Note, the first $R$ location sites in the set $\{1, \ldots, N\}$ will be considered as TCR sites

\section{Parameters:}

$N=$ number of location sites

$M=$ number of fire location sites

$R=$ number of locations sites identified as top corporate risk

$P=$ number of fire stations to be opened (this will be parametrized as a curve versus the coverage percentage). This

helps the decision-maker in a what-if scenario analysis

$w_{i}=$ weight of location $i$

$S_{i}=$ response time target for location $i$ beyond which a location is considered "uncovered"

$a_{i j}=\left\{1\right.$ if response time from fire station $j$ to location $i$ is not greater than $S_{i}$; otherwise 0$\}$

$V=$ maximum response time allowed for secondary coverage

$b_{k j}=\{1$ if response time from fire station $j$ to location $k$ is not higher than $V$; otherwise 0$\}$

$S C=$ Percentage of secondary coverage required

\section{Decision Variables:}

$x_{j}=\{1$ if a fire station $j$ is opened; otherwise 0$\}$

$y_{i}=\{1$ if a location $i$ is covered as a primary coverage; otherwise 0$\}$

$z_{k}=\{1$ if a location $k$ is covered as a secondary coverage; otherwise 0$\}$

\section{Mathematical Model}

The above notations are used to formulate the resultant model, referred to as Weighted Maximum Coverage Location Problem with Secondary Coverage for Top Corporate Risk (WMCLP-STCR) shown below.

$$
\text { Maximize } T=\sum_{i=1}^{N} w_{i} * y_{i}+\sum_{k=1}^{R} w_{k} * z_{k}
$$

Subject to

$\sum_{j=1}^{M} a_{i j} * x_{j}-y_{i} \geq 0, \forall i$ 
$\sum_{j=1}^{M} b_{k j} * x_{j}-z_{k} \geq 1, \forall k$

$\sum_{j=1}^{M} x_{j} \leq P$

$\sum_{k=1}^{R} y_{k}=R$

$\sum_{k=1}^{R=1} z_{k} \geq$

$x_{j}, y_{i} z_{k}=\{0,1\}, \forall i, j$, and $k$

The objective function (Eq. 3.1) aims to maximize the summation of two sub-objectives: the first being the weighted total number of locations covered, and the second is the weighted total of TCR locations covered at least twice. The rationale behind including the second term in the objective function is to ensure the model provides the maximum value of secondary coverage of TCR locations. If the second part of the objective function is not included, then the secondary coverage value will be equal to the value specified by secondary coverage (SC) value in (Eq. 3.6). Therefore, including the second term in the objective will ensure the maximum value is obtained. The constraint (Eq. 3.2) is the usual relationship coverage constraint relating the incident location $\left(y_{i}\right)$ to the fire station $\left(x_{j}\right)$ which ensures that an incident location $i$ can only be covered if there is an open fire station $j$ with $a_{i j}=1$, i.e., it can be reached within the target response time. The constraint (Eq. 3.3) is to ensure secondary coverage of TCR locations, and the greater than equality is used so that at least two fire stations can cover the incident location. Together (Eq. 3.2) and (Eq. 3.3) make sure that the TCR location is primarily and secondarily covered by at least two different fire stations. The constraint (Eq. 3.4) ensures the number of fire stations to be sited does not exceed the number of fire stations specified by the decisionmaker. The constraint (Eq. 3.5) is an additional restriction to cater to the primary coverage of TCR locations. Together constraints (Eq. 3.5) and (Eq. 3.6) ensure that the number of TCR locations covered as primary and secondary coverage by two different fire stations is more than the percentage specified by the decision-maker. The last constraints define the domain of the decision variables.

\subsection{Stochastic Maximum Coverage Location Models}

The model in section 3.1 is deterministic; that is, the response time is assumed to be known and deterministic. When responding to incidents, there might be delays on the way to an incident location, or even before that, the fire station might be busy responding to another incident. Therefore, such realistic considerations must be accounted for in the modeling. Without loss of generality, such considerations are commonly referred to as modeling with uncertainty or stochastic programming. There are different ways with which such models could be solved. Here, we focus on those that assume knowledge of the coverage probability. Namely, we consider the maximum expected covering location model which will explained in detail below. Note, in the numerical section, we shall compare our model with uncertainty with the scenario-based optimization.

In this section, we model the uncertainty using the maximum expected covering location model (MEXCLP) introduced by Daskin (1983). The probability used in Daskin's model assumes independence and uses the same probability for all facilities. In our model, we estimate the facility's primary and secondary coverage probability of each incident location from historical records. Therefore, the variables in Section 3.1 would need to be changed to account for such dependency and the related decision variables. For example, in the following, we need to know whether a location is covered and by which fire station. This is because every couple $i$ and $j$ has a different coverage probability. Firstly, the probability for primary and secondary coverage could be estimated from the response data for each fire station to each location. Secondly, the location variables would need to be modified from one value for a location to multiple values for each fire station and location combination. Both changes will be explained in detail in the below sections.

\section{MEXCLP-STCR Problem formulation}

In this section, we introduce additional or modified notations that are different from the notations presented in Section 3.1.

\section{Parameters:}

$q p_{i j}=$ probability that station $j$ can cover incident location $i$ as a primary coverage $\left(q p_{i j} \leq a_{i j}\right)$

$q s_{k j}=$ probability that station $j$ can cover incident location $k$ as secondary coverage $\left(q s_{i j} \leq b_{i j}\right)$

\section{Decision Variables:}

$y_{i j}=\{1$ if a location $i$ is covered as a primary coverage by fire station $j$; otherwise 0$\}$

$z_{k j}=\{1$ if a location $k$ is covered as a secondary coverage by fire station $j$; otherwise 0$\}$ 


\section{Mathematical Model}

The formulation of MEXCLP-STCR using the above notations gives:

$$
\begin{aligned}
& \text { Maximize } T=\sum_{j=1}^{M} \sum_{i=1}^{N} w_{i} * q p_{i j} * y_{i j}+\sum_{j=1}^{M} \sum_{k=1}^{R} w_{k} * q s_{k j} * z_{k j} \\
& \text { Subject to } \\
& \sum_{j=1}^{M} a_{i j} * x_{j}-\sum_{j=1}^{M} y_{i j} \geq 0, \forall i \\
& \sum_{j=1}^{M} b_{k j} * x_{j}-\sum_{j=1}^{M} z_{k j} \geq 0, \forall k \\
& \sum_{j=1}^{M} x_{j} \leq P \\
& \sum_{j=1}^{M} y_{k j} * x_{j} \geq 1, \forall k \\
& \sum_{j=1}^{M} z_{k j} * x_{j} \geq 2, \forall k \\
& x_{j}, y_{i j}, z_{k j}=\{0,1\}, \forall i, j, k
\end{aligned}
$$

The objective (Eq. 3.8) aims to maximize the sum of two sub-objectives: the first being the weighted score of the locations covered considering the primary coverage probability, and the second is the weighted score of TCR locations secondary covered considering the secondary coverage probability. The constraint (Eq. 3.9) is the usual relationship coverage constraint relating the incident location $\left(y_{i j}\right)$ with the fire station $\left(x_{j}\right)$. The rationale behind using the variable $\left(y_{i j}\right)$ is to incorporate the primary coverage probability for each couple of fire station and incident location. The constraint (Eq. 3.10) together with (Eq. 3.13) ensure the secondary coverage of TCR locations by at least two fire stations. The constraint (Eq. 3.11) ensures the number of fire stations to be sited does not exceed the number of fire stations specified by the decision-maker. (Eq. 3.14) is the usual binary constraints. (Eq. 3.12) and (Eq. 3.13) are not linear, and optimization might not guarantee an optimal solution. Therefore, linearization is required, refer to appendix B for that, which leads to an expansion of the problem size. As we shall show in the following section, the problem size could be substantially reduced leveraging the inherent model properties.

\section{Model Properties}

The model presented by Daskin (1983) had interesting properties at the optimal solution, which are as follow:

$\sum_{j=1}^{M} a_{i j} * x_{j}=\sum_{j=1}^{M} y_{i j}$

$\sum_{j=1}^{M} x_{j}=P$

By exploring the MEXCLP-STCR optimization behaviour, we have noticed several properties of the model presented in this section. These properties are an extension to Daskin's model, which are as follow:
(1) $a_{i j} * x_{j}=y_{i j}, \forall i$ and $j$
(2) $b_{k j} * x_{j}=z_{k j}, \forall k$ and $j$

Clearly, Eq. (3.17) gives Eq. (3.9) and Eq. (3.18) gives Eq. (3.10). The opposite is also true because we have a maximization objective function together with $q p_{i j} \leq a_{i j}$ and $q s_{k j} \leq b_{i j}$. For the above properties, the MEXCLP-STCR model can be reformulated to reduce the number of variables and constraints as follow:

Maximize $T=\sum_{j=1}^{M} \sum_{i=1}^{N} w_{i} * q p_{i j} * a_{i j} * x_{j}+\sum_{j=1}^{M} \sum_{k=1}^{R} w_{k} * q s_{k j} * b_{k j} * x_{j}$ (3.19)

Subject to

$\sum_{j=1}^{M} x_{j} \leq P$

$\sum_{j}^{M} a_{k j} * x_{j} \geq 1, \forall k$

$\sum_{j=1}^{M} b_{k j} * x_{j} \geq 2, \forall k$

$x_{j}=\{0,1\}, \forall j$

Firstly, the $y_{i j}$ and $z_{k j}$ variables are replaced with their equivalents from (Eq. 3.17) and (Eq. 3.18) to yield the objective function (Eq. 3.19). Secondly, (Eq. 3.9) constraint is removed since the resultant constraint will be satisfied with the definition of $a_{i j}$ and $x_{j}$ (i.e., being binary variables). Thirdly, (Eq. 3.12), and (Eq. 3.13) are replaced to yield (Eq. 3.21), and (Eq. 3.22) respectively. For (Eq. 3.22), the right side is used to ensure at least two fire stations can cover TCR location as secondary coverage. From the model properties outlined above, the enhanced formulation has yielded an improved version of the MEXCLP-STR model with $x_{j}$ 's as decision variables. The new formulation yields $2 K+J+1$ constraints compared to the linearized model with $3 K+7 K * J+(J+1) * I+3 J+1$ and $J$ number of 
variables compared to the linearized model with $(J+1) * I+3 K * J$.

\section{Case Study Solution}

To illustrate the benefits of the models presented in the previous sections, a case study at a large company complex is solved. All models were solved using COIN-OR CBC MIP solver (Forrest and Lougee-Heimer, 2005).

\subsection{General data for all models}

For all models, the following parameters and indices are used. The company currently has two existing fire stations (namely fire station A and fire station B), and the company is considering three potential fire station locations (fire station $\mathrm{C}$, fire station $\mathrm{D}$, and fire station $\mathrm{E}$ ). There is a total of 134 incident locations, and 10 of those locations are identified as TCR locations. Therefore, in this case, we have $N=5$ fire stations, $M=134$ locations, $R=10 \mathrm{TCR}$ locations, and $P$ (i.e., number of fire stations to be opened) is parameterized to develop the scenarios for each model. The historical data used is the fire incidents in the last five years totalled 1791 incidents. Four experienced fire professionals at the company were interviewed to perform the pairwise comparison to determine the weight of each TCR location $\left(w_{i}\right)$. The weights for other locations are assumed to be equal and set to one. The summary of pairwise comparison results is shown in Table 4-1.

The relative weights were converted to an absolute weight based on (Eq. 4.1), which looks at the remainder of the division of the relative weights multiplied by a constant and rounds up if the remainder is of more than 0.05 , otherwise mathematical rounding is employed. The rationale behind using this equation is that some facilities importance is quite close, and this formula allows for ranking and does not exacerbate the difference between close values. It is worth noting that the below formula is unique to this paper and has not been found in the published literature. Let $R W_{i}$ denote the relative weight of TCR location $i, C$ denote a constant chosen as 30 by the subject matter experts, and $w_{i}$ denote the absolute weight of $i$.

IF $\left(\left(R W_{i}^{*} \mathrm{C}\right)-\right.$ Integer $\left.\left(R W_{i} * \mathrm{C}\right)\right) /\left(R W_{i}{ }^{*} \mathrm{C}\right)>0.05$ then $w_{i}=\operatorname{round}-u p\left(R W_{i} * \mathrm{C}\right)$

Else $w_{i}=$ round-down $\left(R W_{i}^{*} \mathrm{C}\right)$

(Eq. 4.1)

The response time target for location $i$ depends on the location type where $S_{i}$ is 5 minutes if the location is classified as community, and 10 minutes if it is classified as industrial. The maximum response time target for secondary coverage $(V)$ for all locations is 15 minutes.

\subsection{Case study optimization with WMCLP-STCR}

To assess the impact of increasing the number of fire stations on the coverage, two sets of fire stations are used: the existing fire stations (fire station A and fire station B), and the five fire stations from A to E. Note, any scenario that has less than two fire stations would not be considered for this model since this model requires a minimum of two fire stations for the TCR locations coverage. Before progressing further, the percentage of coverage and weighted coverage calculation are shown in (Eq. 4.2) and (Eq. 4.3), respectively.

Percentage of coverage $=\frac{\sum_{i=1}^{N} y_{i}}{N}$

Percentage of weighted coverage $=\frac{\sum_{i=1}^{N} w_{i} y_{i}}{N}$ 
Table 4-1: Weight $\left(w_{i}\right)$ for Top 10 Corporate Risk

\begin{tabular}{|l|l|l|l|}
\hline Incident Location & $\begin{array}{l}\text { Area } \\
\text { Classification }\end{array}$ & $\begin{array}{l}\text { Relative } \\
\text { Weight }\left(R W_{i}\right)\end{array}$ & $\begin{array}{l}\text { Weight of the } \\
\text { facility }\left(w_{i}\right)\end{array}$ \\
\hline Health Centre & Community & $20.36 \%$ & 6 \\
\hline R\&D Centre & Industrial & $18.80 \%$ & 6 \\
\hline Core Office Area & Community & $16.96 \%$ & 5 \\
\hline Tower Complex & Industrial & $12.44 \%$ & 4 \\
\hline Corporate Data Centre & Industrial & $11.31 \%$ & 4 \\
\hline Aircraft Fuelling Facility & Industrial & $7.03 \%$ & 3 \\
\hline Tanks Farm & Industrial & $5.59 \%$ & 2 \\
\hline Residential Camp M & Community & $3.51 \%$ & 2 \\
\hline Industrial Park & Community & $2.75 \%$ & 1 \\
\hline Equipment and Transportation Area & Community & $1.24 \%$ & 1 \\
\hline
\end{tabular}

To assess the current performance, the WMCLP-STCR model is solved using the existing fire stations, and it resulted in $62.66 \%$ as weighted coverage of all locations; see the first set of bars in Fig 4.1. The TCR locations constraint was relaxed to provide a feasible solution, which resulted in nine TCR locations being covered. Considering the set of all fire stations, two scenarios are possible for the data set used in the model. The first scenario considers only two fire stations, the weighted coverage of all locations is improved to $89.87 \%$ with all TCR locations being covered twice, see the second set of bars in Fig 4.1. The solution set corresponds to fire station D and fire station E. The last scenario has the solution set (A, C, and D) that cover all TCR locations twice, along with providing the highest weighted coverage of $98.10 \%$; see the last set of bars in Fig 4.1. It should be noted that the solution set that provides the maximum primary coverage will be the optimal solution set for the secondary coverage. This logic follows since the primary coverage time is less than that of the secondary coverage.

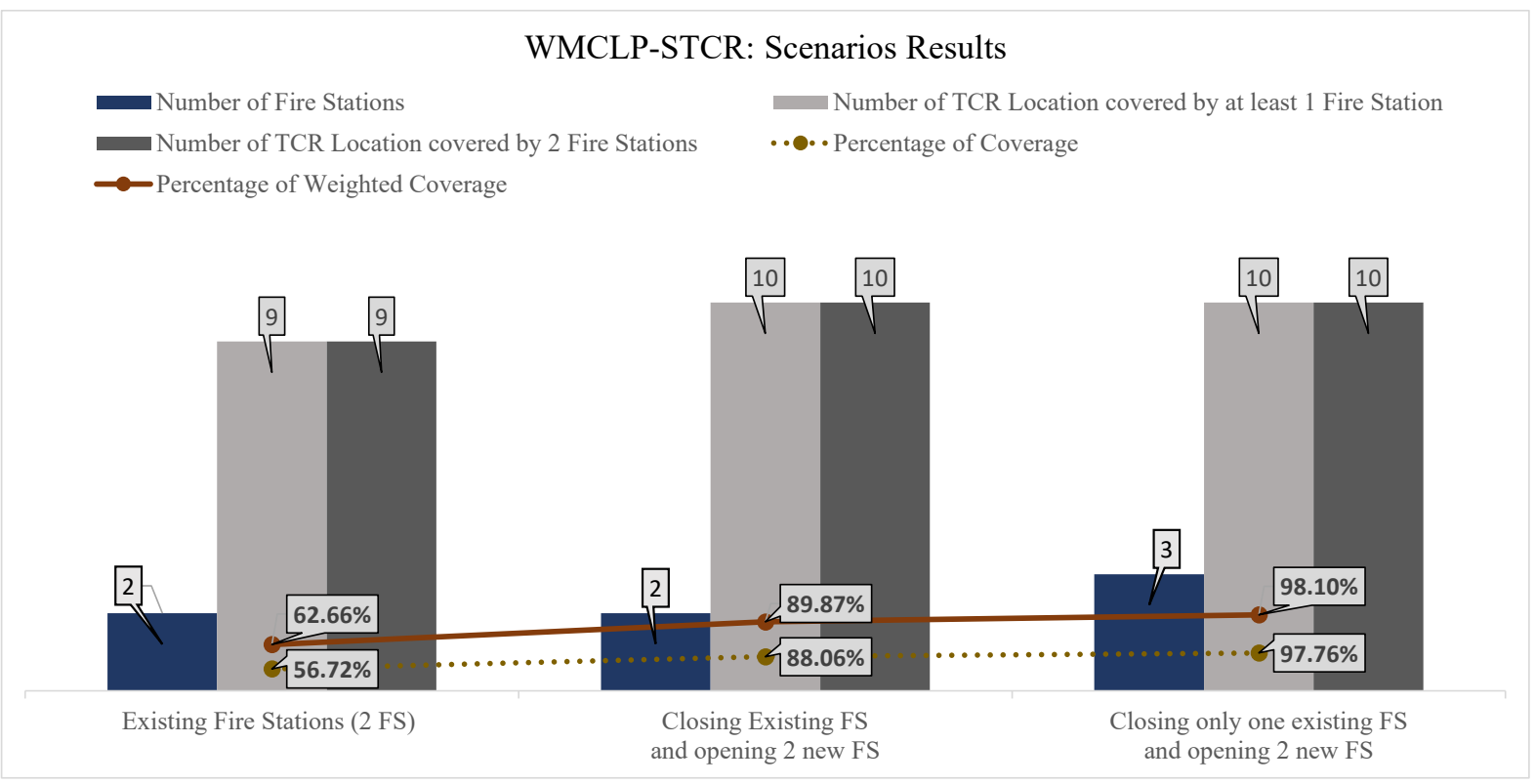

Figure 4.1: WMCLP with TCR Locations being covered scenarios optimization results

\subsection{Case study stochastic optimization with MEXCLP-STCR}

For the MEXCLP, the primary coverage probability $\left(q p_{i j}\right)$ is estimated using normal distribution where the mean is considered as the target response time for each incident type, and the standard deviation is calculated from historical 
records. The use of normal distribution is assumed as it was challenging to fit distribution for each location and fire stations as we must fit 670 distributions (134 locations and five fire stations). The assumption of normality is a better approximation to reality than merely assuming the average response time. The normal distribution can be estimated by two parameters: the mean and the standard deviation. The mean and standard deviation for each location and fire station are calculated from historical records whenever applicable. The rationale behind using the standard deviation from the historical records is to capture the uniqueness of each incident location and the variability in the response provided by the respective fire station. The same logic was applied to the secondary coverage probabilities $\left(q s_{k j}\right)$ with considering the target response time (i.e., mean) as 15 minutes. Like the previous section, the cases are generated for two sets of fire stations: the existing fire stations (fire station A and fire station B), and the five fire stations from A to E. For the current fire stations, the model is infeasible since fire station B cannot cover all TCR locations. It should be noted the interpretation of the weighted coverage percentage is different from the ones presented in section 4.2. This difference is due to incorporating the probability as a function of the fire station and incident locations. To formally state it, the calculation for the percentage of coverage and weighted coverage are shown in (Eq. 4.3) and (Eq. 4.4), respectively.

Percentage of coverage $=\frac{\sum_{j=1}^{M} \Sigma_{i=1}^{N} q p_{i j} y_{i j}}{N}$

Percentage of weighted coverage $=\frac{\sum_{j=1}^{M} \sum_{i=1}^{N} w_{i} * q p_{i j} * y_{i j}}{N}$

Considering the set of all fire stations, three scenarios are possible for the data set used in the model. The first scenario considers only two fire stations; the weighted coverage of all locations is improved to $89.87 \%$, where the solution set corresponds to fire station D and fire station E; see the first set of bars in Fig 4.2. The second scenario is considering opening three new fire stations; the weighted coverage of all locations is improved to $97.47 \%$ and with four TCR locations being covered twice. This scenario corresponds to the solution set (C, D, and E), see the second set of bars in Fig 4.2. This solution set is different when compared to other models, which is attributed to the fact that those fire stations have a relatively higher coverage probability than the other stations. The third scenario considers opening three new fire stations (fire stations $\mathrm{C}, \mathrm{D}$, and $\mathrm{E}$ ) while keeping one of the existing fire stations (i.e., fire station A). This scenario has a $98.10 \%$ weighted coverage, with all TCR locations being covered twice; see the third set of bars in Fig 4.2. The fourth scenario keeps all five fire stations open, which yields the same coverage percentage of $98.10 \%$ as the previous scenario; see the last set of bars in Fig 4.2. It should be noted that the value of the objective function in Eq. 4.4 is different for the last two scenarios, although the weighted coverage is the same. This difference is due to a higher number of stations covering TCR locations under the scenario of five fire stations than that of four fire stations, as shown in Table 4-2.

Table 4-2: Illustration of MEXCLP-STCR Scenarios

\begin{tabular}{|l|l|l|l|l|l|}
\hline Fire station name & A & B & C & D & E \\
\hline Number of TCR Locations covered with 4 fire stations & 2 & - & 5 & 9 & 9 \\
\hline Number of TCR Locations covered with 5 fire stations & 5 & 7 & 9 & 9 & 9 \\
\hline
\end{tabular}

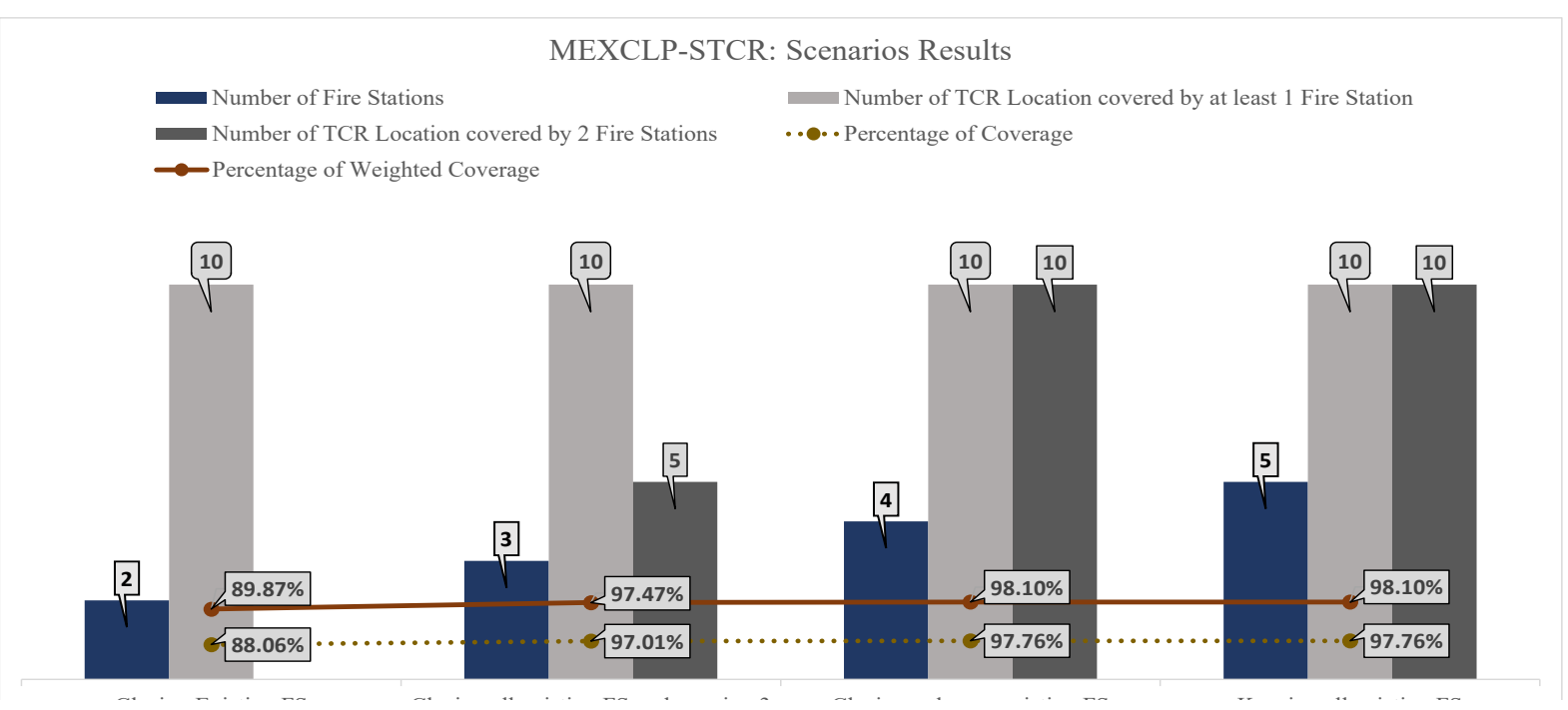

Figure 4.2: MEXCLP with TCR Locations being covered scenarios optimization results

(C) IEOM Society International 
Two secondary measures are introduced to measure the performance of each scenario, which are shown in Fig 4.3. From Fig 4.3, the best solution that meets the company requirement of having all TCR locations being primarily covered by one station and by at least two fire stations as a secondary coverage is a solution set corresponding to fire stations $(\mathrm{C}, \mathrm{D}$, and E). All other solution sets either provide less or more than the company requirements.

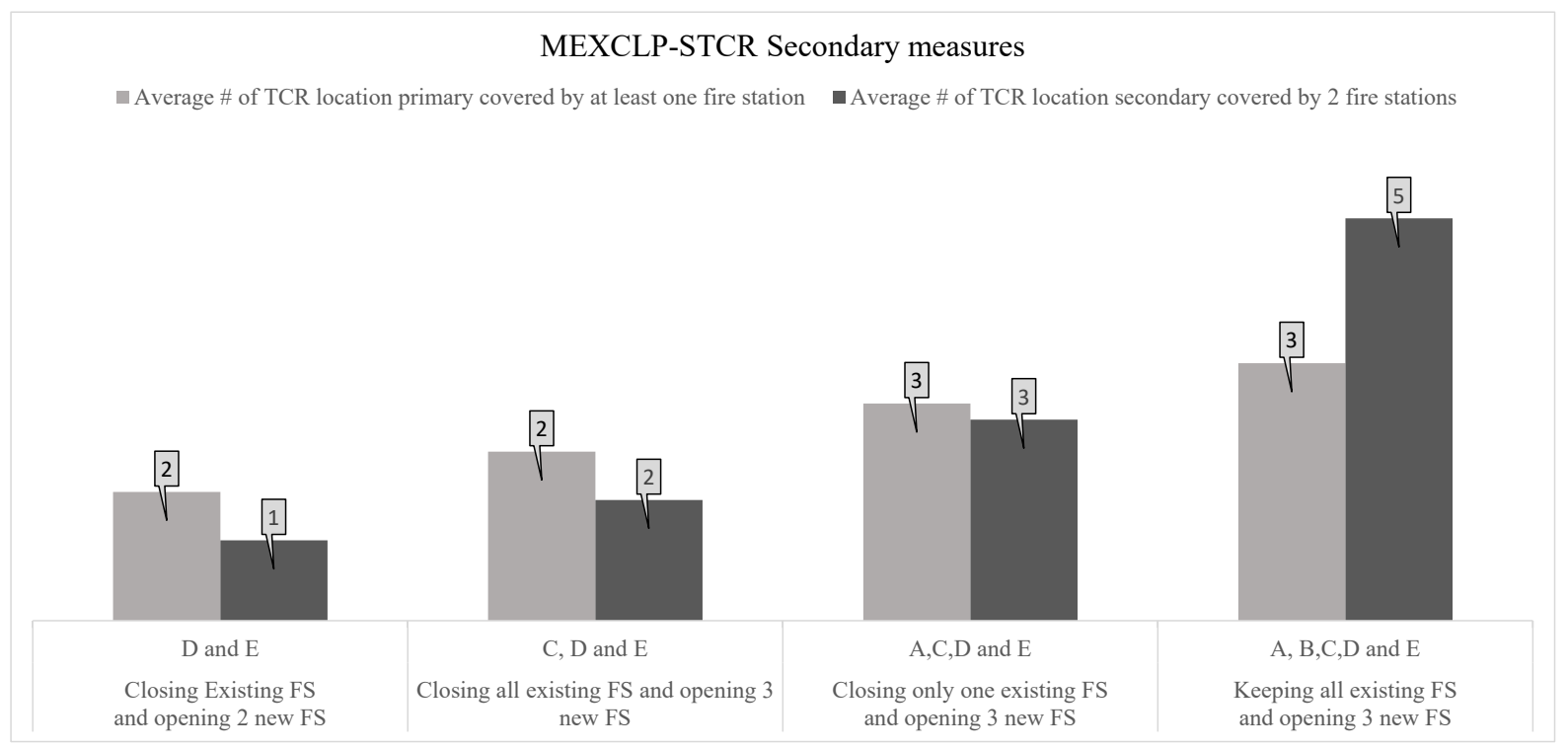

Figure 4.3: MEXCLP with TCR Locations being covered scenarios

\subsection{Case study stochastic optimization with SWMCLP-STCR}

In this section, we shall consider the standard scenario-based optimization of MCLP-STCR with uncertainty, see Appendix A for details. This is done to compare its results with the MEXCLP-STCR results in the previous section. Two input data are needed to model uncertainty using scenario-based optimization. The data sets are the response time and the probability of each scenario. A set of three scenarios (i.e., $U$ is 3 the number of scenarios considered) were used to model the uncertainty in the response time (i.e., primary, and secondary). The first scenario is the average of all records for a given incident location and fire station combination. The second (third) scenario is the best (worst) response time, and wherever applicable, the lowest (highest) response time is considered as the best (worst) time. For cases where the response time is estimated from company maps due to a lack of historical data, the best time is considered $10 \%$ lower ( $25 \%$ higher) than the estimated time. The primary coverage probability of each scenario $\left(q p^{l}\right)$ occurring is determined from historical records, where if the response time of an incident is $10 \%$ less (more) than the average time is considered as the best (worst) time, and elsewhere it is considered as the average time. Then, the primary coverage probabilities can be calculated for each scenario from historical records. For example, the number of incidents reached within the average time is 263 incidents out of 1791 incidents, which results in a primary coverage probability of $14.68 \%$ for the average scenario. Similarly, the secondary coverage probabilities for each scenario $\left(q s^{l}\right)$ are calculated, and the results are summarized in Table 4-3.

Table 4-3: Coverage Probabilities for Average, Best, Worst scenarios

\begin{tabular}{|ll|ll|ll|}
\hline 2. & Scenario & 3. & Primary Coverage Probability & 4. & Secondary Coverage Probability \\
\hline 5. & Average Time & 6. & $14.68 \%$ & 7. & $0.39 \%$ \\
\hline 8. & Best Time & 9. & $51.54 \%$ & 10. $96.82 \%$ \\
\hline 11. & Worst Time & $12.33 .78 \%$ & 13. $2.79 \%$ \\
\hline
\end{tabular}


The secondary coverage probability for a given scenario will be much less than the primary coverage probability because the secondary coverage time (i.e., 15 minutes) is more than the primary coverage time (community $=5$ minutes, industrial $=10$ minutes). Similar to the previous section, the cases are generated for two sets of fire stations: the existing fire stations (fire station A and fire station B) and the five fire stations from A to E. To assess the current performance, the SWMCLP-STCR model is solved using the existing fire stations, and it resulted in $81.16 \%$ weighted coverage, with seven TCR locations being covered twice; see the first set of bars in Fig 4.4. It should be noted that the interpretation of the weighted coverage percentage is different from the ones presented in section 4.3. This difference is due to incorporating the uncertainty over three scenarios (Average time, Best time, Worst time) where the weighted coverage of each scenario is multiplied by the probability of that scenario occurrence and the weighted coverage percentage. To formally state it, the calculation for the percentage of coverage and weighted coverage are shown in (Eq. 4.6) and (Eq. 4.7), respectively.

$$
\begin{aligned}
& \text { Percentage of coverage }=\frac{\sum_{l=1}^{W} q p^{l} \sum_{i=1}^{N} y_{i}^{l}}{N} \\
& \text { Percentage of weighted coverage }=\frac{\sum_{l=1}^{W} q p^{l} \sum_{i=1}^{N} w_{i *} y_{i}^{l}}{N}
\end{aligned}
$$

Considering the set of all fire stations, four scenarios are possible for the data set used in the model. The first scenario is considering only two fire stations, the weighted coverage of all locations is improved to $89.67 \%$ with covering nine TCR locations twice where the solution set corresponds to fire station C and fire station D, see the second set of bars in Fig 4.4. This scenario solution is different from the solution set of the model presented in Section 4.2. This difference is because fire stations $\mathrm{C}$ and $\mathrm{D}$ can uniquely cover 22 locations, whereas fire station $\mathrm{E}$ is dominated by fire station $\mathrm{D}$ in almost all the scenarios. The second scenario has the same solution set as of the previous section since the solution set (A, C, and D) can cover all TCR locations twice, along with providing $92.13 \%$ as weighted coverage, see the third set of bars in Fig 4.4. Due to incorporating uncertainty, more fire stations would need to be opened to maximize the weighted coverage across all scenarios, shown in the third and fourth scenarios. The third scenario corresponds to $93.84 \%$ as weighted coverage, see the fourth set of bars in Fig 4.4. The solution set calls for opening three new fire stations (fire stations $\mathrm{C}, \mathrm{D}$, and $\mathrm{E}$ ) while keeping one of the existing fire stations (i.e., fire station $\mathrm{A}$ ). The reason for incorporating the fire station $\mathrm{E}$ is that under the worst-case scenario two non-TCR locations and one TCR location can be uniquely covered by fire station E. The scenarios thus far cannot cover all TCR locations since under the worst-case scenario the maximum number of TCR locations that can be covered is 9 . The fourth scenario corresponds to keeping all existing fire stations and opening three new fire stations with a slight improvement to the weighted coverage percentage to be $94.69 \%$; see the last set of bars in Fig 4.4. The solution provides a slight improvement, which is attributed to the fact that fire station B can uniquely cover two locations of which one is a TCR location. A compromise must be made here as it is not economically feasible to have all five fire stations opened. The solution set corresponding to fire stations (A, C, D) seems reasonable as it covers all TCR locations as primary coverage, with nine TCR locations being secondarily covered by at least two fire stations.

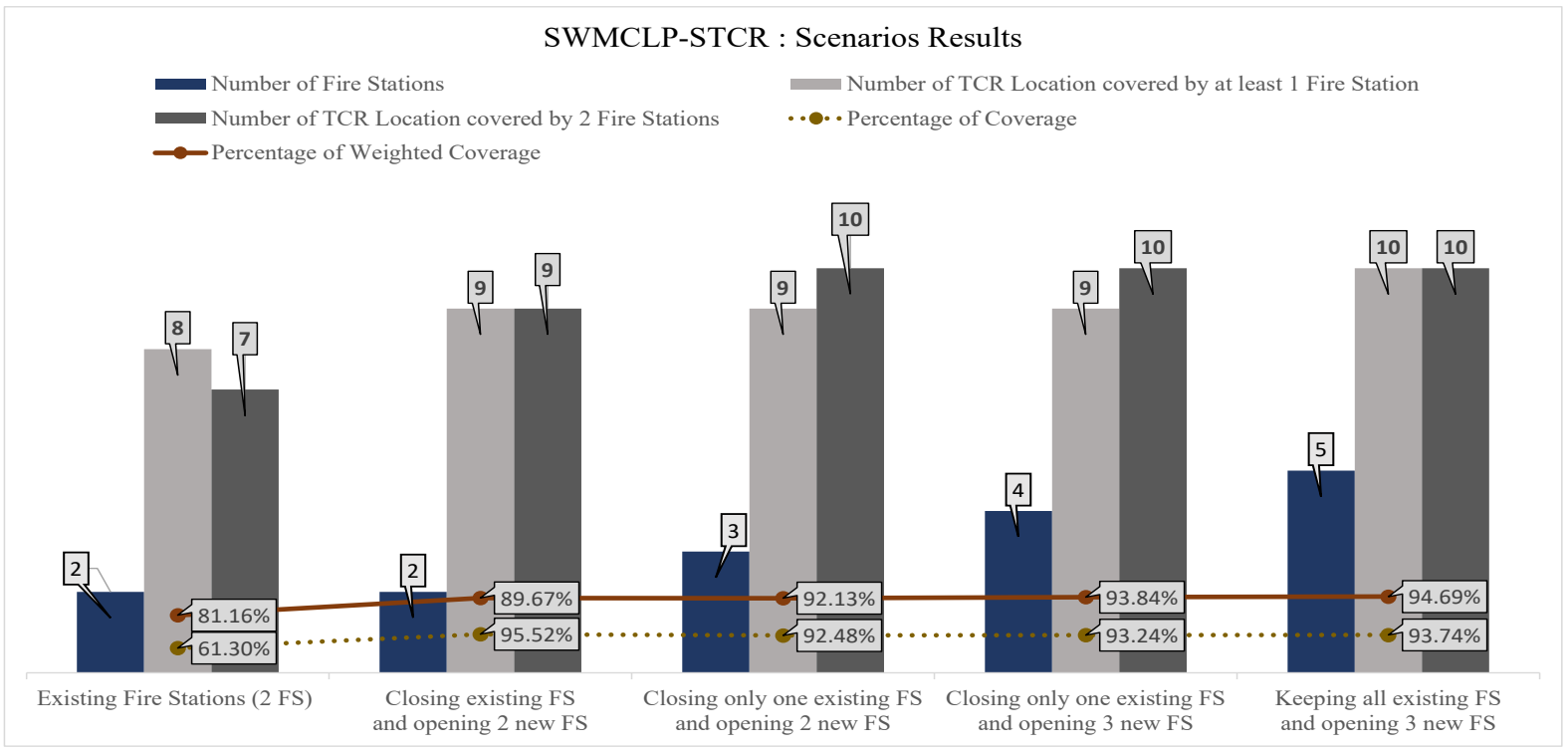

Figure 4.4: SWMCLP with TCR Locations being covered scenarios 
Due to uncertainty, two secondary measures are introduced to measure the scenarios performance, shown in Figure 4.5. The first measure is the average number of fire stations covering a TCR location as primary coverage, and the second is the average number of fire stations covering a TCR location as secondary coverage. From Figure 4.5, the best solution that meets the company requirement of having all TCR locations being primarily covered by one station and at least by two fire stations as a secondary coverage is the solution set corresponding to fire stations (A, C, D). All other solution sets either provide less or more than the company requirements.

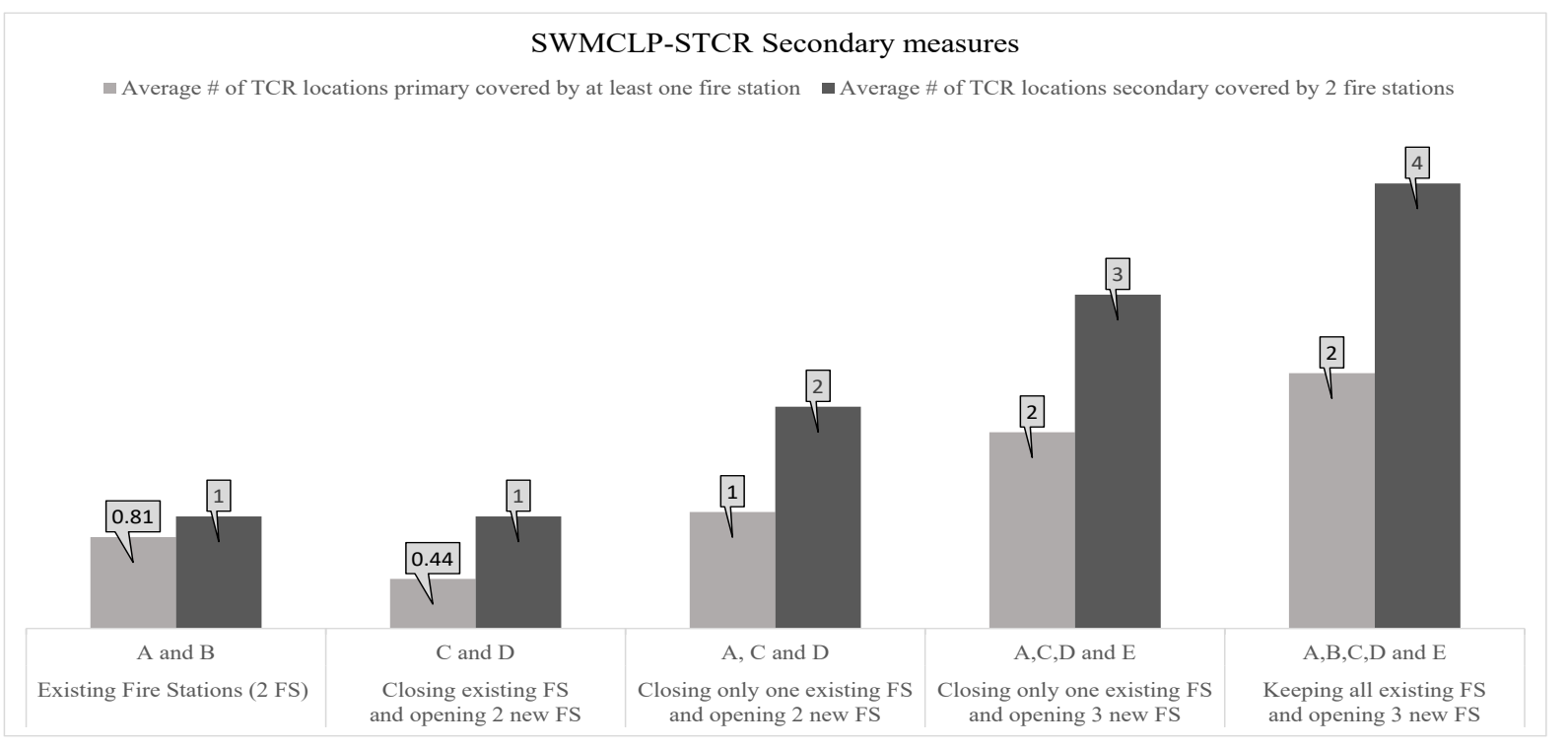

Figure 4.5: SWMCLP-STCR Secondary measures

\section{Results Discussion}

Although there are several studies on coverage problems in the literature, the models presented in this paper have new and unique features. Namely, the company requires to ensure secondary coverage with secondary response time for the TCR locations. Also, the assumption of independent coverage probability is relaxed to have the probability as a function of the facility site and the incident location. This relaxation is needed as it can be hard to satisfy the independence assumption in practice. From the models' results shown in the previous sections, Fire station D was included in all the models when considering one or two fire stations. Also, Fire station B was proven to be improperly located, as evident by all scenarios considered. Therefore, it is recommended to close fire station $\mathrm{B}$ and consider keeping fire station $\mathrm{D}$ in the set of the fire stations to be opened. Fire station E should be considered next as it has proven to have better coverage with fire station D as shown in the first and third models. In all scenarios of three fire stations, fire station C and D performed relatively better than other fire stations and are part of all solution sets. This is also supported by the fact that fire station $\mathrm{C}$ and $\mathrm{D}$ cover a total of 22 locations combined that are not covered by the other stations (see Table 5-1).

To realize the value of incorporating uncertainty, a comparison is made between the solution set of the deterministic model (WMCLP-STCR) and the stochastic models MEXCLP-STCR and SWMCLP-STCR. For a valid comparison, the same solution set needs to be considered for the same scenario. For SWMCLP-STCR, we chose the solution set corresponding to fire stations $\mathrm{D}$ and $\mathrm{E}$ to illustrate the value of incorporating uncertainty. (Eq. 4.7) is used to compare the two solutions; the stochastic solution for fire Station D and E shows $89.21 \%$ compared to the deterministic solution shows $89.87 \%$. The comparison shows a decrease of $0.66 \%$, which is expected since the deterministic case considered only the average response time. For MEXCLP-STCR, we chose the solution set corresponding to fire stations A, C, and $\mathrm{D}$ as an illustration for the value of incorporating uncertainty. (Eq. 4.5) is used to compare the two solutions; the stochastic solution for the selected solution set shows $64.03 \%$ compared to the deterministic solution of $98.10 \%$. The comparison shows a $34.70 \%$ decrease, which is expected since the deterministic case assumes a coverage probability of one for the fire station and location combination. On the other hand, the stochastic case considers a coverage probability of less than one, and only two locations out of all locations covered have a coverage probability of one. The uncertainty impacted the coverage and provided the decision-maker with realistic performance measures to evaluate the fire station's performance. 
Table 5-1: Summary of fire stations' performance measures

\begin{tabular}{|l|l|l|l|l|l|}
\hline $\begin{array}{l}\text { Performance } \\
\text { Measures }\end{array}$ & Fire Station A & Fire Station B & Fire Station C & Fire Station D & Fire Station E \\
\hline $\begin{array}{l}\text { Number of } \\
\text { Locations } \\
\text { Covered }\end{array}$ & 61 & 35 & 78 & 109 & 80 \\
\hline $\begin{array}{l}\text { Locations } \\
\text { where only one } \\
\text { station can } \\
\text { cover }\end{array}$ & 0 & 0 & 8 & 14 & 0 \\
\hline
\end{tabular}

Based on the previous results, we show the ability of mathematical programming to solve coverage problems of fire departments in urban areas efficiently. We explicitly included the coverage uncertainty in the modelling to generate a robust solution that meets the strict company requirements.

\section{Conclusion}

This paper considers a real case study of a large company in Saudi Arabia to locate the right number of fire stations. The aim is to maximize the weighted coverage for all locations and maximize the number of covered top risk corporate locations (TCR) locations. The case study provides several natural extensions to existing models such as Maximum Covering Location Problem (MCLP) and Maximum Expected Covering Location Problem (MEXCLP) to incorporate realistic business decisions (i.e., the criticality of the location to the company). In this paper, a deterministic mathematical model and an approach to deal with uncertainty in the coverage data were proposed. The deterministic model includes two extensions. The first is ensuring TCR locations are covered, and the second is allowing secondary coverage for those critical locations. The second model that deals with uncertainty is an extension in two ways to the MEXCLP. The first is to relax the assumption of the independent probability of MEXCLP to have the probability as a function of the facility site and the incident location. The second is to incorporate that TCR locations are to be covered by at least two fire stations. The mains contributions are summarized as follows. We extend the maximum coverage location problem (MCLP) to account for TCR locations covered by the fire stations as primary coverage and secondary coverage. We extend the Maximum Expected Covering Location Problem (MEXCLP) to have the probability of coverage as a function of the fire station and the incident location. As well as we provide an efficient formulation for the extended MEXCLP model. Finally, we applied the proposed model to a real case study consisting of five fire stations, 134 incident locations, 10 TCR locations, and using five years of fire incidents data. The results showed the model ability to efficiently generate a robust solution that takes the coverage uncertainty into account.

In this paper, we were limited to coverage problems of fire departments from a modelling and application perspective. Future research scope can include other applications such as emergency care service and emergency repair in maintenance service. The incorporation of a fire station mobilization plan for each type of incident and truck deployment for each fire station is also interesting to consider. From a theoretical side, this work could be extended by considering a multi-objective model that minimizes the network cost, average and maximum response times while maintaining the targeted coverage level.

\section{References}

Abdul Ghani, N., \& Ahmad, N. (2017). Analysis of MCLP, Q-MALP, MQ-MALP with travel time uncertainty using Monte Carlo simulation. Journal of Computational Engineering, 2017, 1-15.

Aktaş, E., Özaydin, Ö., Bozkaya, B., Ülengin, F., \& Önsel, Ş. (2013). Optimizing fire station locations for the Istanbul Metropolitan Municipality. Interfaces, 43(3), 240-255.

Aytug, H., \& Saydam, C. (2002). Solving large-scale maximum expected covering location problems by genetic algorithms: a comparative study. European Journal of Operational Research, 141(3), 480-494.

Çatay, B. (2011). Siting new fire stations in Istanbul: A risk-based optimization approach. OR Insight, 24(2), 77-89.

Church, R., \& ReVelle, C. (1974). The maximal covering location problem. Papers of the Regional Science Association, 32(1), 101-118.

Cordeau, J. F., Furini, F., \& Ljubić, I. (2019). Benders decomposition for very large-scale partial set covering and maximal covering location problems. European Journal of Operational Research, 275(3), 882-896. 
Daskin, M. S. (1983). Maximum expected covering location model: formulation, properties, and heuristic solution . Transportation Science, 17(1), 48-70.

Daskin, M. S., Hogan,1999 K., \& Revelle, C. (1988). Integration of multiple, excess, backup, and expected covering models. Environment \& Planning B: Planning \& Design, 15(1), 15-35.

Davari, S., Fazel Zarandi, M. H., \& Hemmati, A. (2011). Maximal covering location problem (MCLP) with fuzzy travel times. Expert Systems with Applications, 38(12), 14535-14541.

Dreyfuss, M., \& Giat, Y. (2017). Optimal spares allocation to an exchangeable-item repair system with tolerable wait. European Journal of Operational Research, 261(2), 584-594.

Erkut, E., Ingolfsson, A., \& Erdogan, G. (2008). Ambulance location for maximum survival. Naval Research Logistics, 55(1), 42-58.

Farahani, R. Z., Asgari, N., Heidari, N., Hosseininia, M., \& Goh, M. (2012). Covering problems in facility location: A review. Computers and Industrial Engineering. Elsevier Ltd.

Forrest, J., \& Lougee-Heimer, R. (2005). CBC user guide. In Emerging theory, methods, and applications (pp. 257-277). INFORMS.

Hogan, K., \& ReVelle, C. (1986). Concepts and Applications of Backup Coverage. Management Science, 32(11), 14341444.

Ingolfsson, A., Budge, S., \& Erkut, E. (2008). Optimal ambulance location with random delays and travel times. Health Care Management Science, 11(3), 262-274.

Karasakal, O., \& Karasakal, E. K. (2004). A maximal covering location model in the presence of partial coverage. Computers and Operations Research, 31(9), 1515-1526.

Megiddo, N., Zemel, E., \& Hakimi, S. L. (1983). The Maximum Coverage Location Problem. SIAM Journal on Algebraic Discrete Methods, 4(2), 253-261.

Murray, A. T. (2016). Maximal Coverage Location Problem: Impacts, Significance, and Evolution. International Regional Science Review, 39(1), 5-27.

Narasimhan, S., Pirkul, H., \& Schilling, D. A. (1992). Capacitated emergency facility siting with multiple levels of backup. Annals of Operations Research, 40(1), 323-337.

National Fire Protection Association (2020) NFPA 1710, standard for the organization and deployment of fire suppression operations https://www.nfpa.org/ codes-and-standards/all-codes-and-standards/list-of-codes-andstandards/detail?code $=1710$

Ozbaygin, G., Yaman, H., \& Karasan, O. E. (2016). Time constrained maximal covering salesman problem with weighted demands and partial coverage. Computers and Operations Research, 76, 226-237.

Pirkul, H., \& Schilling, D. (1989). The capacitated maximal covering location problem with backup service. Annals of Operations Research, 18(1), 141-154.

Shapiro, A., \& Philpott, A. (2007). A tutorial on stochastic programming. Manuscript. Retrieved from http://stoprog.org/stoprog/SPTutorial/TutorialSP.pdf

Toregas, C., Swain, R., ReVelle, C., \& Bergman, L. (1971). The Location of Emergency Service Facilities. Operations Research, 19(6), 1363-1373.

Ulander, A. (2015). Optimization Based Decision Support Tools for Fire and Rescue Resource Planning. Optimization Based Decision Support Tools for Fire and Rescue Resource Planning. Linköping University Electronic Press.

van den Berg, P. L., Kommer, G. J., \& Zuzáková, B. (2016). Linear formulation for the Maximum Expected Coverage Location Model with fractional coverage. Operations Research for Health Care, 8, 33-41.

\section{Appendix A: Weighted Stochastic Maximum Coverage Location Problem with Secondary Coverage for Top Corporate Risk using Scenario-Based Optimization (SWMCLP-STCR)}

The model presented in Section 3.1 can be extended to account for uncertainty with exploring the possible scenarios based on the decision maker objective and data availability. Clearly, the number of scenarios will impact the problem size and the solution feasibility; therefore, such considerations will need to be assessed during the problem formulation (see section 4.4). Fundamentally, the optimization in this model is solving the model presented in Section 3.1 several times (one for each response time realization) and seeking the optimal solution across all response time realizations. In addition to the notations presented in Section 3.1, the below notations will be used to formulate the stochastic problem using scenario-based optimization.

Indices:

$l=$ index of scenarios considered (where $l=1 \ldots U)$

Parameters:

$U=$ number of scenarios considered

$a_{i j}^{l}=\left\{1\right.$ if response time from station $j$ to location $i$ is not greater than $S_{i}$ in scenario $l$; otherwise 0$\}$ 
$b_{k j}^{l}=\{1$ if response time from station $j$ to location $k$ is not greater than $V$ in scenario $l$; otherwise 0$\}$

$q p^{l}=$ primary coverage probability in scenario $l$

$q s^{l}=$ secondary coverage probability in scenario $l$

\section{Decision Variables:}

$y_{i}^{l}=\{1$ if a location $i$ is covered as a primary coverage under scenario l; otherwise 0$\}$

$z_{k}^{l}=\{1$ if a location $k$ is covered as a secondary coverage under scenario l; otherwise 0$\}$

Using the above notations, the formulation of SWMCLP-STCR is given below:

Maximize $T=\sum_{l=1}^{U} q p^{l} \sum_{i=1}^{N} w_{i} * y_{i}^{l}+\sum_{l=1}^{U} q s^{l} \sum_{k=1}^{R} w_{k} * z_{k}^{l} \quad$ (A.1)

Subject to

$\sum_{j=1}^{M} a_{i j}^{l} * x_{j}-y_{i}^{l} \geq 0, \forall i$ and $l$

$\sum_{j=1}^{M} b_{k j}^{l} * x_{j}-z_{k}^{l} \geq 1, \forall k$ and $l$

$\sum_{j=1}^{M} x_{j} \leq P$

$\sum_{k=1}^{R} y_{k}^{l}=R, \forall l$

$\sum_{k=1}^{R} z_{k}^{l} \geq S C * R, \forall l$

$x_{j}, y_{i}^{l}, z_{k}^{l}=\{0,1\}, \forall i, j, k$, and $l$

The objective (Eq. A.1) aims to maximize the summation of two sub-objectives: the first being the total weighted score of locations covered across all scenarios, and the second is the weighted total of TCR locations covered twice across all scenarios. For each objective, the value of the total weighted score of the scenario will be multiplied with the coverage probability, primary or secondary, of that scenario. As noted previously, the optimization in this model is solving the model presented in Section 3.1 using response time realizations considered in the scenarios and optimizing across all these scenarios. The constraint (Eq. A.2) is the usual relationship coverage constraint relating the incident location $i$ under scenario $l\left(y_{i}^{l}\right)$ with the fire station $\left(x_{j}\right)$. The constraint (Eq. A.3) is to ensure secondary coverage of TCR locations under each scenario, and the greater or equal equality is used so that at least two fire stations can cover the incident location. The constraint (Eq. A.4) ensures the number of fire stations to be sited does not exceed the number of fire stations specified by the decision-maker. The constraint (Eq. A.5) is an additional restriction to ensure primary coverage of all TCR locations under each scenario. The constraint (Eq. A.6) ensures the number of TCR locations covered twice (i.e., primary, and secondary) under each scenario is more than the specified secondary coverage by the decision-maker. Effectively, the optimization will provide different results depending on the secondary coverage value specified. The last constraint is the usual binary constraints.

\section{Appendix B: Linearized Model of MEXCLP-STCR}

To linearize (Eq. 3.12), we need to introduce a few notations which are as follow:

1. A new decision variable is added to account for the multiplication of the two variables (i.e., $y_{k j} * x_{j}$ ) which is defined as follows: $t_{k j}=\{1$ if a fire station $j$ can cover location $k$; otherwise 0$\}$

2. We need to ensure the variable is equal to or greater than the lower bound specified by the relation between the opened facility and a covered location by that facility

$t_{k j} \geq y_{k j}-\left(1-x_{j}\right) * M M, \forall k$ and $j$

3. The variable should be less than or equal to the upper bound (i.e., location $k$ is covered by fire station $j$ ).

$t_{k j} \leq y_{k j}, \forall k$ and $j$

4. The total coverage of each TCR location should be more than one to ensure each fire station is only opened if it covers a TCR location.

$\sum_{j}^{M} t_{k j} \geq 1, \forall k$

5. The total coverage of each fire station should be chosen to cover the most of TCR locations

$\sum_{k}^{R} t_{k j} \leq x_{j} * M M, \forall j$

6. The normal binary constraint applies here since $t_{k j}$ is bounded by a linear combination of two binary variables; therefore, any realization should be one or zero.

$t_{k j}=\{0,1\}, \forall k$ and $j$

Similarly, to linearize (Eq. 3.13), we need to introduce few notations which are as follow:

1. A new decision variable is added to account for the multiplication of the two variables (i.e., $z_{k j} * x_{j}$ ) which is defined as follow: $O_{k j}=\{1$ if a fire station $\mathrm{j}$ can cover location $\mathrm{k}$; otherwise 0$\}$

2. We need to ensure the variable is equal to or greater than the lower bound specified by the relation between the opened facility and a covered location by that facility 
$O_{k j} \geq z_{k j}-\left(1-x_{j}\right) * M M, \forall k$ and $j$

3. The variable should be less than or equal to the upper bound that is a location $\mathrm{k}$ is covered by fire station $\mathrm{j}$.

$O_{k j} \leq z_{k j}, \forall k$ and $j$

4. The total coverage of each TCR location should be more than one. That is to ensure each fire station is only opened if it covers a TCR location.

$\sum_{j}^{M} O_{k j} \geq 1, \forall k$

5. The total coverage of each fire station should be chosen to cover the most of TCR locations

$\sum_{k}^{R} O_{k j} \leq x_{j} * M M, \forall j$

6. The normal binary constraint applies here since $O_{k j}$ is bounded by a linear combination of two binary variables. Therefore, any realization should be one or zero.

$O_{k j}=\{0,1\}, \forall k$ and $j$

Subsequently, the linear formulation is shown below:

Maximize $T=\sum_{j=1}^{M} \sum_{i=1}^{N} w_{i} * q p_{i j} * y_{i j}+\sum_{j=1}^{M} \sum_{k=1}^{R} w_{k} * q s_{k j} * z_{k j}$

Subject to

$\sum_{j=1}^{M} a_{i j} * x_{j}-\sum_{j=1}^{M} y_{i j} \geq 0, \forall i$

$\sum_{j=1}^{M} b_{k j} * x_{j}-\sum_{j=1}^{M} z_{k j} \geq 0, \forall k$

$\sum_{j=1}^{M} x_{j} \leq P$

$t_{k j} \geq y_{k j}-\left(1-x_{j}\right) * M M, \forall k$ and $j$

$t_{k j} \leq y_{k j}, \forall k$ and $j$

$\sum_{j=1}^{M} t_{k j} \geq 1, \forall k$

$\sum_{k=1}^{R} t_{k j} \leq x_{j} * M M, \forall j$

$O_{k j} \geq z_{k j}-\left(1-x_{j}\right) * M M, \forall k$ and $j$

$O_{k j} \leq z_{k j}, \forall k$ and $j$

$\sum_{j=1}^{M} O_{k j} \geq 2, \forall k$

$\sum_{k=1}^{R} O_{k j} \leq x_{j} * M M, \forall j$

$x_{j}, y_{i j}, z_{k j}, t_{k j} O_{k j}=\{0,1\}, \forall i, j, k$

The constraints specified by (Eq. B.15), (Eq. B.16), (Eq. B.17), and (Eq. B.18) are the linearized constraints to cater for TCR locations being covered as primary coverage. The constraints specified by (Eq. B.19), (Eq. B.20), (Eq. B.21), and (Eq. B.22) are the linearized constraints to ensure the number of TCR locations covered twice. The last constraints are the usual binary constraints. The rest of the constraints and variables are unchanged from the model presented in section 3.1 .

\section{Biographies}

Abdulaziz S. Alzahrani is currently working as Organization Performance Advisor at Saudi Aramco and has more than 9 years of total professional experience in various fields from Strategy and Organization design, Business Process Improvement, and Management Consulting. Abdulaziz earned his bachelor's degree in Industrial \& Systems Engineering from King Fahad University of Petroleum \& Minerals (KFUPM) in January 2013 and completed his master's degree in December 2019 from the same institution. He has also won the "Excellence in Academic Research" award in 2018 and 2019 at the Annual KFUPM Student Forum. Abdulaziz highlights his Professional Interests as Data Analytics, Workforce Optimization and Modelling, and Operations Research.

Dr. Ahmad Al Hanbali received his Phd in 2006 from University of Nice, France. Between 2007 and 2017, he was faculty in the department of Industrial engineering at Univeristy of Twente, The Netherlands. He is a full member of the International Society of Inventory Research (ISIR), Production and Operations Management Society (POMS). He is a former member of the Beta research school The Netherlands, Twente is Maintenance Excellence (TIME), Service Logistics Forum (SLF), and Dutch Network on the Mathematics of Operations Research (LNMB). He is a member of many technical program committees of international conferences. He served as a reviewer for several international journals. 